\title{
Weighting method of the Fourier-kinoform synthesis
}

\author{
A.V. Kuzmenko \\ International Center "Institute of Applied Optics", NAS of Ukraine, \\ 10G, Kudryavskaya str., 04053 Kyiv,Ukraine, e-mail: avk@iao.kiev.ua
}

\begin{abstract}
A new iterative Fourier transform method of synthesis of kinoforms is presented. Two object-depended filters (an amplitude filter and a phase one) are used in the object plane on the iterative calculation of a kinoform instead of a single (phase) filter as usual. The amplitude filter is a system of weight coefficients that vary in the process of iterations and control the amplitude of an input object. The advantages of the proposed method over other ones are confirmed by computer-based experiments. It is found that the method is most efficient for binary objects.
\end{abstract}

Keywords: digital holography, holographic optical elements, phase retrieval, phase-only elements.

Manuscript received 18.06.08; accepted for publication 20.06.08; published online 30.09.08.

\section{Introduction}

There exists a high number of iterative algorithms aimed at the solution of phase problems, including the problem of a kinoform. The majority of algorithms was developed on the basis of the so-called error-reduction (ER) algorithm [1]. It was refined in works by Fienup $[2,3]$ on solving the problems of star interferometry (a group of input-output algorithms). I mention also the subsequent works, e.g., by Wyrowski and Bryngdahl [4], Wyrowski [5], and Yang, Gu [6] in which the iterative algorithms were further improved. At the present time, all they form a family of the so-called iterative Fourier transform (IFT) algorithms. The comparative analysis of a number of algorithms from this family is available in some surveys (see, e.g., [7, 8]).

It is known that processing the amplitude of a field (A-field) in the spectral plane is the same in all IFT algorithms of calculation of a kinoform. This operation is nonlinear and consists in the reduction of the A-field to unity. The algorithms differ from one another by a mean of processing the field in the object plane which depends on the final purpose, namely, on a function which should be realized by a kinoform (the beam splitting, beam shaping, image generation, and so on). It is worth to note that, despite the diversity of operations used in the object plane, all they, are linear (except the ER-algorithm).

\section{Algorithm}

In the present work, I give a modified ER-algorithm of calculation of a kinoform, whose main unique distinction from the classical ER-algorithm [1] consists in the use of a new nonlinear operation [9] of processing the A-field in the object plane. This operation in its complex-valued version was used earlier by us in the synthesis of doublephase holograms [10]. To clarify its application, the work of the algorithm is illustrated in Fig. 1. First, one or several iterations $\left(K_{\text {er }}\right)$ are realized by the classical ERscheme which requires no explanations. Then, in all iteration with $k>K_{\text {er }}$ on the formation of an input, $f$ will be replaced by a new function defined as

$f_{k}=\alpha_{k} f$,

where the weight coefficients $\alpha_{k}$ are determined by the recurrence relation

$\alpha_{k}=\alpha_{k-1} \beta_{k-1}(k>1)$,

$\beta_{k-1}=f /\left(\left|g_{k-1}\right|+\varepsilon\right)$.

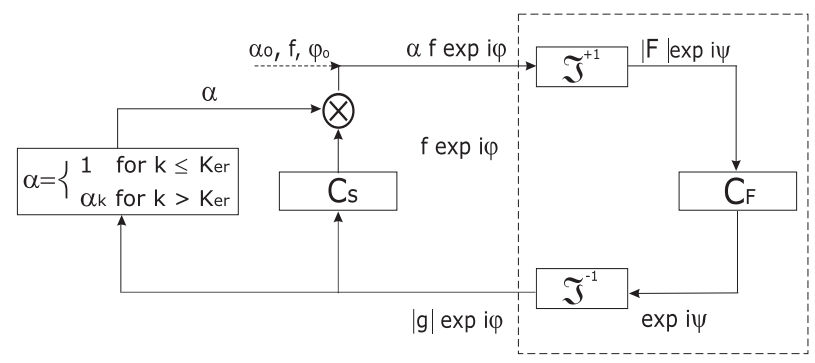

Fig. 1. Weighting IFT algorithm.

Here, $\left|g_{k-1}\right|$ is the reconstructed amplitude on the $(k-1)$ th iteration, and $\varepsilon$ is a small number $\sim 10^{-10}$, excluding the division by zero. It should be mentioned that $f$ is real. Operations (1)-(3) are heuristic and have no strict mathematical justification. The physical sense of 
the coefficients $\alpha_{k}$ becomes clear if the block of the algorithm separated by a dashed line in Fig. 1 is considered, according to Fienup [3] as a nonlinear unit with the input $\alpha f \exp (i \varphi)$ output $g$, and action operator $\mathscr{F}^{+1} C_{\mathbf{F}} \mathscr{F}^{-1}$. From the viewpoint of the theory of imageprocessing system, the coefficients $\alpha$ is nothing but the collection of coefficients of a negative feedback "outputinput"': if the amplitude $\left|g_{k-1}\right|$ on the $(k-1)$ th iteration at some point $\left(x^{\prime}, y^{\prime}\right)$ of the plane of images is more than a given value $f$, then, on the next $k$ th iteration, the input $f$ at the corresponding point $(x, y)$ will be corrected. Namely, it will be decreased by $\alpha_{k}$ times, and vice versa. At the same time, from the viewpoint of optics, the system of coefficients $\alpha_{k}$ normalized to unity can be interpreted as some object-depended amplitude filter which acts on the initial object $f$ and varies in the process of iterations. It is clear that, for all ER-iterations, $\alpha(x, y)$ $=\alpha_{0}(x, y)=1(x, y)$.

\section{Experiment}

A number of model experiments with various objects was realized with the purpose to study the potentialities of the method. For the sake of comparison, analogous experiments were also performed with the use of a kinoform version of the input-output (IO) algorithm by Fienup [3]. In all the cases, the same phase starting diffuser with a uniform distribution of phases in the interval $0-2 \pi$ is used. The variance of the amplitudes of images reconstructed in the process of iterations was evaluated using the formula

$$
\sigma_{g}(k)=\frac{\sum_{l, m}\left(f_{l, m}-\mu(k)\left|g_{l . m}(k)\right|\right)^{2}}{\sum_{l, m}\left(f_{l, m}\right)^{2}},
$$

where $\quad \mu(k)=\left(\frac{\sum_{i, j}\left(f_{i, j}\right)^{2}}{\sum_{i, j}\left|g_{i, j}(k)\right|^{2}}\right)^{1 / 2}$

is the scale factor, the indices $l, m$ and $i, j$ run over the points, where the amplitude of an initial object $f$ is nonzero, and $k$ is the iteration number.

In the experiments involving the IO algorithm, the optimum value of the object-depended coefficient $\beta=\beta_{\text {opt }}$ in the equations for the input function (see Eqs (8) and (9) in Ref. [3]) is used by attaining the best behavior of the function $\sigma_{g}(k)$ during iterations in all the cases. The value of $\beta_{\text {opt }}$ was determined by means of the cyclic repetition of the procedure of synthesis for various values of $\beta$ (from the interval 0.1-5.0 with a step of 0.1).

In Figs 2 to 4, the results of model experiments on the synthesis of the kinoforms of binary and halftone objects with a dimension of $64 \times 64$ counts are presented.
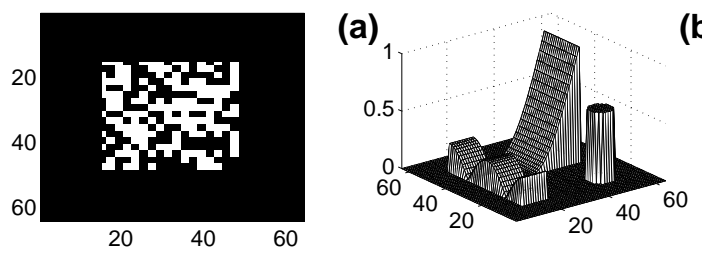

(b)

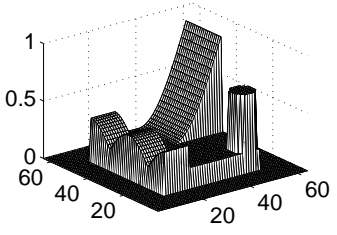

(c)

Fig. 2. Objects 64×64: (a) binary; (b), (c) halftone without and with a base (equal 0.17).

\subsection{Binary object}

In Fig. 3, the plots characterizing the quality of the image of a binary object (Fig. 2a) reconstructed by a kinoform are presented. As seen from Fig. 3a, the weighting algorithm allows one to decrease the dispersal of the one-bit-intensity $\Delta I_{\text {one-bits }}$ given by the ERalgorithm practically to zero, i.e., the algorithm does not reveal the effect of stagnation relative to binary objects. In our example, 180 weighting-iterations reduce $\Delta I_{\text {one-bits }}$ from 0.008 to $7.6 \times 10^{-7}$ (Fig. 3b), whereas 1500 such iterations result in $\Delta I_{\text {one-bits }}=2 \times 10^{-12}$.
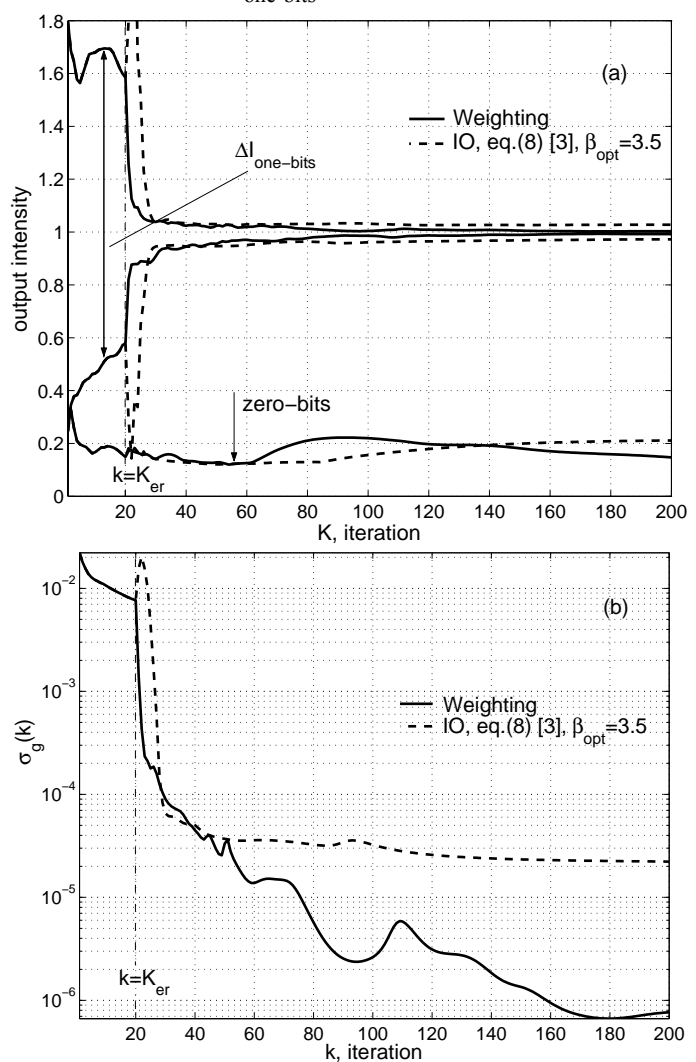

Fig. 3. The kinoform of the binary object (Fig. 2a): (a) range of output intensities, (b) variance of amplitude of the reconstructed images $v s$ iteration number. 
At the same time, the IO algorithm (with optimized $\beta)$ "stops" at the value $\Delta I_{\text {one-bits }}=2.5 \times 10^{-5}$. That is, it falls in a minimum of $\sigma_{g}(k)$ which is quite deep, but, nevertheless, is local. As for the ratios of the minimum one-bits-intensity to the zero-bits intensity for three algorithms, they are equal, respectively, to 4 (ER), 4.53 (IO), and 7 (weighting). Figure $3 \mathrm{~b}$ demonstrates the effect of diminution of the variance $\sigma_{g}(k)$ on the transition from one algorithm to another one. Analogous results were obtained also for other binary objects with dimensions of $64 \times 64$ and $128 \times 128$.

\subsection{Halftone object}

A somewhat more complicated situation is observed for halftone objects, one of which is presented in Fig. 2b. As was shown by model experiments, the kinoforms of such objects calculated with the help of the weighting algorithm reconstruct a high-quality image only in the range of amplitudes from $\sim 0.15$ to unity (on the normalization of the image to unity). The rest amplitudes are distorted to a variable degree. The proper reconstruction of all the amplitudes, including those close to zero, can be reached if the initial object is positioned on a pedestal (Fig. 2c) whose height is $\sim 15$ $20 \%$ of its maximum amplitude and if the reconstructed image amplitude (the intensity in an optical experiment) is cut off by the pedestal level. It is obvious that, in this case, the useful diffraction efficiency of a kinoform decreases. The dependences of $\sigma_{g}$ on the iterations for both compared algorithms given in Fig. 4 indicate that, in the case where a base is supplemented to an object, the weighting algorithm begins to surpass the IO algorithm after a certain number of iterations. In our example with the object in Fig. 2c, the advantage of the weighting algorithm begins to manifest itself after 90 iterations (see Fig. 4), and is characterized almost by the six-order difference by the 1500 th iteration $\left(1 \times 10^{-12}\right.$ against $9.5 \times 10^{-6}$ ). But if the base is absent, the IO algorithm has some advantage.

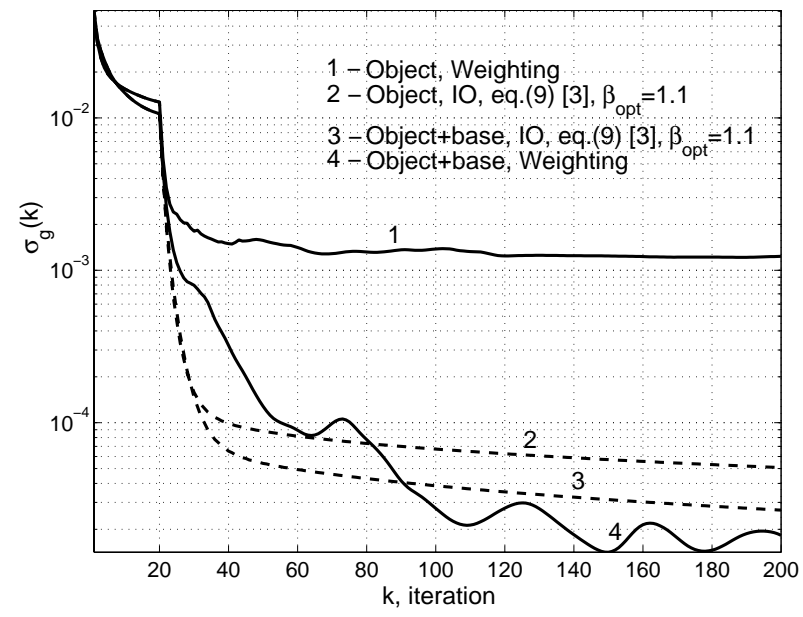

Fig. 4. Variance of amplitude of the reconstructed images for halftone object without and with a base (Fig. 2b, c).
The calculated efficiencies of kinoforms (in parentheses, the values obtained within the IO algorithm are given) are as follows: $91.39(91.25) \%$ for the object in Fig. 2a, 94.82 (92.48) \% for the object in Fig. 2b, and 96.91 (95.02) \% for the object in Fig. 2c.

In the course of calculations, the criticality of the weighting algorithm with respect to a value of the parameter $\varepsilon$ in the formula (3) is verified. By varying $\varepsilon$ from $1 \times 10^{-22}$ to $1 \times 10^{-6}$, the deviation of $\sigma_{g}(\varepsilon)$ from $\sigma_{g}\left(\varepsilon_{10}\right)=10^{-10}$ is determined by the formula

$\Delta_{\sigma}=100 \%\left[\sigma_{g}(\varepsilon)-\sigma_{g}\left(\varepsilon_{10}\right)\right] / \sigma_{g}\left(\varepsilon_{10}\right)$

for various objects with the number of iterations equal to 50 . On the average, $\Delta_{\sigma}$ was $(0.002-0.05) \%$. Thus, the variation of $\varepsilon$ in the indicated limits did not influence practically the exactness of the calculation of a kinoform and, at the same time, excluded the situation where one should divide the numerator in formula (3) by zero.

\subsection{Super-Gaussian (SG) beam shaping}

Within the weighting and IO algorithms, the calculations of kinoforms that are the transducers of the intensity of a Gauss beam of the form $\exp \left[-\left(x^{2}+y^{2}\right) / 2 r_{0}{ }^{2}\right]$ in a SG beam of the form $\exp \left[-\left(x^{\prime 2} / 2 r_{0}{ }^{\prime 2}\right)^{\mathrm{M}}-\left(y^{\prime 2} / 2 r_{0}{ }^{\prime 2}\right)^{\mathrm{M}}\right]$ are performed, where $r_{0}$ and $r_{0}{ }^{\prime}$ are the inflection radii of the Gauss curves, and $\mathrm{M}$ is the $\mathrm{SG}$ order (as known, the calculation of a kinoform involves the square root of the both indicated intensities). In Fig. 5, the input $\left(r_{0}=70\right)$ and the output $\left(r_{0}^{\prime}=25\right)$ intensity profiles for $\mathrm{M}=4$ and $\mathrm{M}=100$ with a dimension of the object (kinoform) of $256 \times 256$ counts are presented. The iteration process with $K_{\text {er }}=10$ was truncated at the 100 th iteration. With regard for the separation of the working part of a SG beam so as shown in Fig. 5, the intensity variance $\sigma_{I}$ and the output efficiency $\eta$ are as follows: $\sigma_{I}=2.9 \times 10^{-4}\left(6.59 \times 10^{-4}\right), \eta=$ $96.46(89.72) \%$ for $\mathrm{M}=4 ; \sigma_{I}=3.7 \times 10^{-5}\left(1.98 \times 10^{-4}\right), \eta=$ $93.63(91.2) \%$ for $\mathrm{M}=100$. The calculation of $\sigma_{I}$ was performed by a formula analogous to (4), but for intensities.

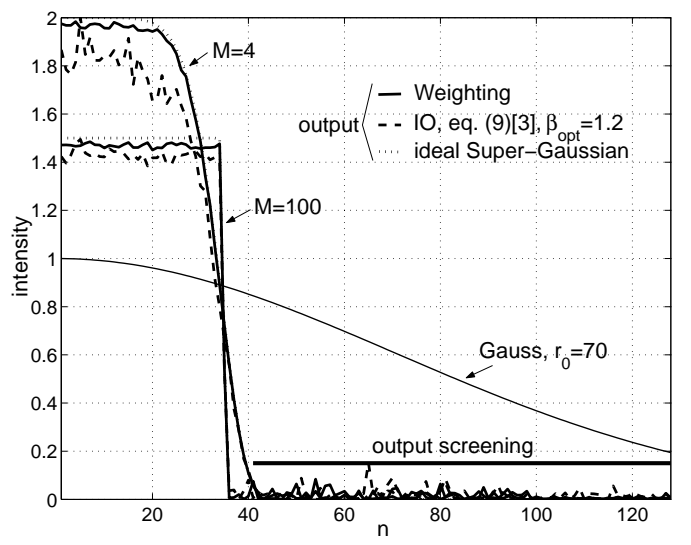

Fig. 5. The profiles of intensities of super-Gaussian beams with $r_{0}{ }^{\prime}=25$ of the 4 th and 100 th orders obtained by calculations of the $256 \times 256$ kinoform within the weighting and IO algorithms. Curves for $\mathrm{M}=4$ and $\mathrm{M}=100$ are vertically shifted up for clarity. 

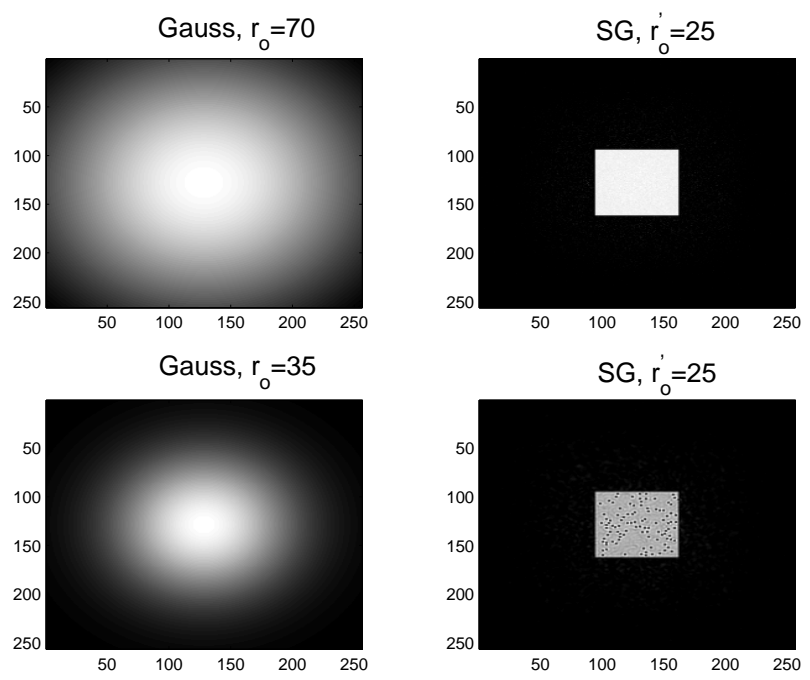

Fig. 6. Proper $\left(r_{0}=70\right)$ and erroneous $\left(r_{0}=35\right)$ choices of the effective width of an illuminating beam on the calculation of the kinoform-former of a super-Gaussian $\left(r_{0}{ }^{\prime}=25\right)$. In the second case, the cross-section of a super-Gaussian is covered by a speckle.

I note that, in the calculation of a kinoform-former of a SG, a special attention should be paid to a choice of $r_{0}$ defining the effective width of a beam illuminating the kinoform. For small $r_{0}$, the kinoform is illuminated by a narrow Gauss beam, which means the actual nullification of light amplitudes on the edges of the kinoform. This is equivalent to a reduction of the band of space frequencies forming a SG. As a result, the pattern of a SG will be covered by a speckle irrespective of the value of $r_{0}{ }^{\prime}$ (see Fig. 6). In more details, the problem of restriction of the frequency band and its relation to the quality of images are considered in [4].

\section{Conclusions}

Thus, the weighting algorithm has high efficiency in the synthesis of the kinoforms of binary objects. It is basically important that the effect of stagnation of the algorithm is absent in this case, i.e., the one-bits variance $\sigma_{g}(k)$ in a reconstructed image tends to zero with increase in the number of iterations and the noise level (zero-bits) is the same as that of other algorithms or lower. The weighting algorithm is also efficient in calculations of kinoforms as the formers of SG laser beams. It must be emphasized that the weighting algorithm contains no parameters requiring the optimization (like $\beta$ in the IO algorithm), which essentially accelerates the counting rate. However, it is expedient to apply this algorithm to halftone objects only in the case where the minimum amplitude of the normalized distribution of an object is $\sim 0.15-0.20$.

\section{References}

1. R.W. Gerchberg and W.O. Saxton, A practical algorithm for the determination of phase from image and diffraction plane pictures // Optik 35, p. 237-246 (1972).

2. J.R. Fienup, Phase retrieval algorithms: a comparison // Appl. Opt. 21, p. 2758-2769 (1982).

3. J.R. Fienup, Iterative method applied to image reconstruction and to computer-generated holograms // Opt. Eng. 19, p. 297-305 (1980).

4. F. Wyrowski and O. Bryngdahl, Iterative Fourier transform algorithm applied to computer holography // J. Opt. Soc. Amer. A 5, p. 1058-1065 (1988).

5. F. Wyrowski, Diffractive optical elements: iterative calculation of quantized, blazed phase structures // J. Opt. Soc. Amer. A 7, p. 961-969 (1990).

6. G.-Z. Yang and B.-Y. Gu, Theory of the amplitudephase retrieval in any linear transform system and its applications // Intern. J. Mod. Phys. B 7, p. 3153-3224 (1993).

7. O. Ripoll, V. Kettunen, and H. P. Herzig, Review of iterative Fourier transform algorithms for beam shaping applications // Opt. Eng. 43, p. 2549-2556 (2004).

8. M. Škeren, I. Richter, and P. Fiala, IterativeFourier-transform algorithm: comparizon of various approaches // J. Mod. Opt. 49, p. 18511870 (2002).

9. A.V. Kuzmenko, UA Patent No. 65295 (priority from 03.07.2003), Bull. 1 (16 January 2006).

10. A.V. Kuzmenko and P.V. Yezhov, Iterative algorithms for off-axis double-phase computergenerated holograms implemented with phase-only spatial light modulators // Appl. Opt. 46, p. 73927400 (2007). 